\title{
AVALIAÇÃO DA REAÇÃO FOTO-FENTON NA DECOMPOSIÇÃO DE RESÍDUOS DE CARRAPATICIDA
}

\author{
Caio Fernando Gromboni e Antônio Gilberto Ferreira \\ Departamento de Química, Universidade Federal de São Carlos, CP 676, 13560-970 São Carlos - SP, Brasil \\ Marcos Yassuo Kamogawa \\ Departamento de Ciências Exatas, Escola Superior de Agricultura “Luiz de Queiroz", Universidade de São Paulo, \\ Piracicaba - SP, Brasil \\ Ana Rita de Araujo Nogueira* \\ Embrapa Pecuária Sudeste, São Carlos, CP 339, 13560-970 São Carlos - SP, Brasil
}

Recebido em 17/6/05; aceito em 5/5/06; publicado na web em 26/9/06

\begin{abstract}
EVALUATION OF THE PHOTO-FENTON REACTION IN THE DECOMPOSITION OF TICK RESIDUES. Experimental procedures based on factorial design and surface response methodology were applied to establishe experimental conditions for the decomposition of a 1:400 (v/v) Supocade ${ }^{\circledast}$ (chlorfenvinphos $13.8 \%$ and cypermethrin 2.6\%) solution, used to control cattle ticks. Experiments exploring photo-oxidative reactions were performed with and without UV radiation, fixing exposition time and pesticide volume, and varying the oxidant mixture. The use of $3.6 \mathrm{mmol} \mathrm{L}^{-1} \mathrm{Fe}^{2+}$ plus $1.9 \mathrm{~mol} \mathrm{~L}^{-1} \mathrm{H}_{2} \mathrm{O}_{2}$ plus $\mathrm{UV}$ radiation provided destruction of $94 \%$ of the original carbon content and reduction of aromatic, aliphatic and carbinolic compounds, evaluated by determination of residual carbon content by ICP OES and NMR analysis.
\end{abstract}

Keywords: photo-Fenton; pesticide residue; UV radiation.

\section{INTRODUÇÃO}

O emprego de defensivos químicos, fertilizantes e sais minerais, tem propiciado a obtenção de produtividades cada vez maiores na agropecuária. Contudo, para atingir tal eficiência, existe o problema ambiental originado pelas elevadas quantidades de resíduos químicos gerados. Nesse contexto, pode ser citada como exemplo a contaminação de solos e águas por pesticidas empregados no controle de carrapatos em bovinos, onde comumente centenas de litros de solução residual de carrapaticida são gerados e muitas vezes descartados indiscriminadamente pelo produtor rural. Durante as aplicações de carrapaticidas em bovinos, são utilizados cerca de 4 a 5 L dessa solução para cada animal adulto. Em regiões em que a pecuária possui grande produção, o volume de solução residuária gerado é expressivo.

Alternativa cada vez mais utilizada para tratamento de resíduos e para decomposição de amostras orgânicas, os processos fotooxidativos são relativamente simples e bastante eficientes. Esses processos baseiam-se na formação de radical hidroxila (HO•), agente altamente oxidante e reativo, com uma grande variedade de classes de compostos, promovendo sua total decomposição ${ }^{1,2}$. Geralmente consistem na união da radiação UV com substâncias químicas que facilitam a produção das hidroxilas, responsáveis pela decomposição dos compostos orgânicos. Diversas substâncias podem ser empregadas com esse objetivo, sendo que algumas se destacam, tais como $\mathrm{TiO}_{2}, \mathrm{H}_{2} \mathrm{O}_{2}, \mathrm{Fe}^{2+}-\mathrm{Fe}^{3+}, \mathrm{O}_{3}$ e $\mathrm{K}_{2} \mathrm{~S}_{2} \mathrm{O}_{8}$. A radiação $\mathrm{UV}$, com comprimentos de onda de 6 a $400 \mathrm{~nm}$, divide-se em ultravioleta próximo, distante e no vácuo. A energia gerada por essa radiação é suficiente para a remoção de elétrons da camada de valência e, conseqüentemente, capaz de romper algumas ligações químicas. Apesar da radiação UV conseguir gerar radicais oxidantes, em muitos casos, principalmente quando o composto não sofre fotólise direta, ou essa é muito pequena, o uso combinado com outros agentes oxidantes propicia um aumento de eficiência.

*e-mail: anarita@cppse.embrapa.br
O emprego de processos fotooxidativos foi utilizado com sucesso no tratamento de resíduos de biocidas ${ }^{2,3}$, de corantes ${ }^{4}$ e de resíduos laboratoriais ${ }^{5}$. Dentre suas principais vantagens podem ser citadas: a mineralização do poluente e não somente sua transferência de fase; a possibilidade de emprego em compostos refratários a outros tratamentos; a transformação de produtos refratários em compostos biodegradáveis; podem ser usados com outros processos (pré e póstratamento); o forte poder oxidante, com cinética de reação elevada; geralmente não há necessidade de pós-tratamento ou disposição final; em muitos casos, há menor consumo de energia, permitindo a redução de custos e, é possível o tratamento in situ .

Em função da baixa toxicidade, fácil obtenção e do baixo custo dos reagentes, a geração de radical hidroxila a partir da reação do ferro com o peróxido de hidrogênio é freqüentemente utilizada como alternativa para tratamento de resíduos. Essa reação foi proposta originalmente por Henry J. H. Fenton, em 1894, para a oxidação do ácido tartárico e é atualmente conhecida como "reação Fenton". A combinação de peróxido de hidrogêncio e íons $\mathrm{Fe}^{2+}$ ou $\mathrm{Fe}^{3+}$ com radiação ultravioleta (UV), denominado processo foto-Fenton ${ }^{7-9}$, produz mais radicais hidroxilas quando comparado ao método Fenton convencional $\left(\mathrm{Fe}^{2+} \mathrm{com} \mathrm{H}_{2} \mathrm{O}_{2}\right)$ ou à simples fotólise, aumentando a eficiência na degradação de poluentes orgânicos, atribuída principalmente à foto-redução de $\mathrm{Fe}^{3+}$ para $\mathrm{Fe}^{2+10}$.

$\mathrm{FeOH}^{2+}+h \mathrm{v} \rightarrow \mathrm{Fe}^{2+}+\mathrm{HO}$

Durante a reação, ocorre também a formação de outros compostos intermediários ${ }^{11}$ : estados excitados da matéria orgânica dissolvida, peróxido de hidrogênio, oxigênio singlete, elétron hidratado, íons superóxidos, radicais organoperóxidos, radicais hidroxila e radicais halogênios. A formação desses radicais é o ponto chave do procedimento, melhorando a eficiência de decomposição.

De maneira geral, durante a reação foto-Fenton podem ser citadas as seguintes etapas do mecanismo: fotorredução de íons $\mathrm{Fe}^{3+}$ formando íons $\mathrm{Fe}^{2+}$, que reagem $\operatorname{com~} \mathrm{H}_{2} \mathrm{O}_{2}$; aumento da decomposi- 
ção de $\mathrm{H}_{2} \mathrm{O}_{2}$ pela absorção da luz UV e fotólise de complexos orgânicos de $\mathrm{Fe}^{3+}$ gerados durante a decomposição. Além destes fatores, a fotólise de hidroxo-complexos de $\mathrm{Fe}^{3+}$ é uma fonte adicional de $\mathrm{HO}$, conforme a reação:

$$
\begin{aligned}
& \mathrm{H}_{2} \mathrm{O}_{2}+\mathrm{Fe}^{2+} \rightarrow \mathrm{Fe}^{3+}+\mathrm{HO}^{-}+\mathrm{HO} \bullet \\
& \mathrm{HO} \bullet+\mathrm{Fe}^{2+} \rightarrow \mathrm{Fe}^{3+}+\mathrm{HO}^{-} \\
& \mathrm{HO} \bullet+\mathrm{RH} \rightarrow \mathrm{H}_{2} \mathrm{O}+\mathrm{R} \bullet \\
& \mathrm{O}_{2}+\mathrm{UV} \rightarrow 2 \mathrm{O}+\mathrm{O}_{2} \rightarrow \mathrm{O}_{3} \\
& \mathrm{O}_{3}+\mathrm{H}_{2} \mathrm{O}+\mathrm{UV} \rightarrow \mathrm{H}_{2} \mathrm{O}_{2}+\mathrm{O}_{2}
\end{aligned}
$$

O planejamento fatorial é uma importante ferramenta estatística e seu uso é importante na química analítica para diferentes amostras e propósitos ${ }^{12-15}$, pois possibilita a visualização das possíveis interações entre as variáveis selecionadas, considerando todos os parâmetros experimentais envolvidos.

O principal enfoque desta proposta foi avaliar a eficiência da decomposição de resíduo de carrapaticida pelo processo foto-Fenton, para uso no tratamento de resíduos orgânicos relativamente concentrados, provenientes da atividade agropecuária. Uma das conseqüências do desenvolvimento do trabalho foi a obtenção de grande quantidade de informações a partir de dados numéricos e a aplicação da quimiometria facilitou a interpretação de forma mais rápida e eficiente dos dados originados.

\section{PARTE EXPERIMENTAL}

\section{Instrumentação}

Para a degradação dos resíduos de solução carrapaticida foi empregada uma câmara fotooxidativa construída em madeira com as dimensões apresentada na Figura 1. Uma lâmpada germicida de ultravioleta de $45 \mathrm{~cm}$ com $15 \mathrm{~W}$ de potência, alimentada por um transformador eletrônico de 110/220 V (normalmente utilizado para lâmpadas fluorescentes) foi instalada dentro da câmara, com um sistema de ajuste de altura para aproximar ou afastar a lâmpada da base onde eram colocadas as amostras. Neste trabalho, a distância foi fixada em $35 \mathrm{~mm}$. O interior da câmara foi revestido com papel alumínio, visando maior aproveitamento da radiação UV a partir da reflexão da luz.

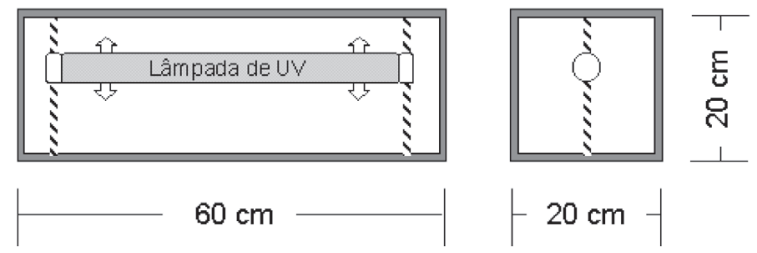

Figura 1. Câmara de irradiação ultravioleta. Construída em madeira e interior revestido em alumínio. Potência da lâmpada $-15 \mathrm{~W}, 45 \mathrm{~cm}$ de comprimento. Maiores informações no texto

Os estudos foram conduzidos a partir da transferência de $3 \mathrm{~mL}$ de solução carrapaticida (1:400 v/v), que apresenta $\mathrm{pH}$ de aproximadamente 3, para frascos de polipropileno. Em função das dimensões da câmara e dos frascos de amostra, 20 recipientes eram tratados simultaneamente.

Os teores de carbono residual (CCR) foram empregados como parâmetro para medida da eficiência da degradação e foram determinados por espectrometria de emissão óptica com plasma indutivamente acoplado (ICP OES, Vista RD, Varian, Multigrave, Austrália) equipado com câmara ciclônica e nebulizador concêntrico, operado em condições robustas com potência de $1,3 \mathrm{~kW}$ e vazão do gás de nebulização de $0,60 \mathrm{~L} \mathrm{~min}^{-1}$, e foi utilizada a linha espectral 193,027 $\mathrm{nm}^{16}$.
Para obtenção dos espectros de ressonância magnética nuclear foi utilizado um equipamento de 9,4 Tesla (400 MHz para freqüência do hidrogênio), marca Bruker, modelo Avance, à temperatura de $298 \mathrm{~K}$.

\section{Reagentes e soluções}

Todas as soluções foram preparadas com reagentes de grau analítico e os volumes finais ajustados com água destilada e deionizada. A vidraria utilizada foi cuidadosamente lavada com detergente e a seguir, para se evitar a contaminação pelo ferro e auxiliar a oxidação de possíveis resíduos orgânicos, foi descontaminada durante 24 h em banho oxidante, contendo $10 \%$ v/v $\mathrm{HNO}_{3}$.

Curvas analíticas foram construídas a partir de soluções estoque contendo $20.000 \mathrm{mg} \mathrm{L}^{-1}$ de C (Merck). Para a degradação das soluções carrapaticidas foram utilizados $\mathrm{FeSO}_{4} .7 \mathrm{H}_{2} \mathrm{O}$ e $\mathrm{H}_{2} \mathrm{O}_{2} 30 \%$ $\mathrm{m} / \mathrm{v}$ (Merck). Nas análises de RMN foram utilizados éter dietílico, clorofórmio $\left(\mathrm{CDCl}_{3}\right)$, ácido fosfórico, tetrametilsilano (TMS) e dimetilformamida (DMF) (Merck).

\section{Resíduo}

O carrapaticida empregado é denominado comercialmente Supocade ${ }^{\circledR}$, concentrado e emulsionável, contendo em cada litro 25 g de Cipermetrina (alfa-ciano-3-fenoxibenzil-2-dimetil-3 (2,2diclorovinil)-ciclopropano carboxilato) e como princípio ativo majoritário $138 \mathrm{~g}$ de Clorfenvinfos (2-cloro-1-(2,4-diclorofenil)-vinildietil-fosfato), sendo considerado de grau de toxidez I (extremamente tóxico). Soluções carrapaticidas foram preparadas simulando condições reais de um banho carrapaticida na proporção de 1:400 (concentrado:água).

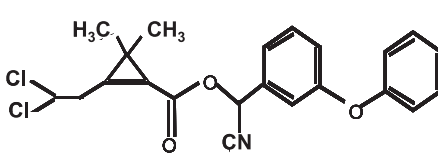

Cipermetrina

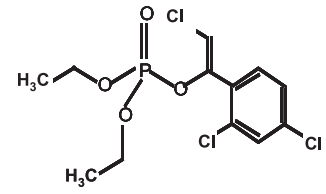

Clorfenvinfos
Figura 2. Fórmulas estruturais do princípio ativo do carrapaticida

\section{Decomposição dos resíduos de carrapaticida}

Para avaliação da eficiência de decomposição a partir das medidas dos teores de carbono residual por ICP OES, foi montado um planejamento fatorial $2^{3}$, onde eram executados experimentos sistemáticos em triplicatas, utilizando soluções contendo $\mathrm{Fe}^{2+}$ na concentração de $1000 \mathrm{mg} \mathrm{L}^{-1}$ com volume mínimo de $1 \mathrm{~mL}$ e máximo de $3 \mathrm{~mL}$ e $\mathrm{H}_{2} \mathrm{O}_{2} 30 \% \mathrm{~m} / \mathrm{v}$, utilizado como oxidante, nos volumes equivalentes aos do $\mathrm{Fe}^{2+}$. O último parâmetro investigado foi a ausência ou a presença da radiação UV, durante um período de $24 \mathrm{~h}$. $\mathrm{O}$ teor de carbono original foi quantificado antes das fotooxidações e os teores de carbono residual ${ }^{16}$ determinados após correção do volume das soluções para $10 \mathrm{~mL}$ com água, imediatamente após a realização das fotooxidações.

Após terem sido estabelecidas as condições analíticas para a utilização da câmara fotooxidativa, 20 novos tratamentos foram avaliados de acordo com as seguintes condições:

a) $3 \mathrm{~mL}$ de solução carrapaticida $+1 \mathrm{~mL}$ de $\mathrm{Fe}^{2+}+1 \mathrm{~mL} \mathrm{de} \mathrm{H}_{2} \mathrm{O}_{2}$ (Fenton);

b) $3 \mathrm{~mL}$ de solução carrapaticida $+24 \mathrm{~h}$ de irradiação UV (UV);

c) $3 \mathrm{~mL}$ de solução carrapaticida $+1 \mathrm{~mL}$ de $\mathrm{Fe}^{2+}+24 \mathrm{~h}$ de irradiação UV (Fe+UV); 
d) $3 \mathrm{~mL}$ de solução carrapaticida $+1 \mathrm{~mL} \mathrm{de} \mathrm{Fe}^{2+}+1 \mathrm{~mL}$ de $\mathrm{H}_{2} \mathrm{O}_{2}+$ $24 \mathrm{~h}$ de irradiação UV (foto-Fenton).

\section{Preparo da amostra para análise de RMN}

As amostras submetidas aos tratamentos descritos anteriormente foram analisadas por RMN, com a finalidade de se verificar as características dos produtos formados após a degradação. A extração líquido-líquido com emprego de éter dietílico foi empregada para a extração dos compostos resultantes. As fases orgânicas foram coletadas e rotoevaporadas em temperatura de $25{ }^{\circ} \mathrm{C}$, sendo a seguir liofilizadas. Na sequiência, foram analisadas por RMN utilizando $\mathrm{CDCl}_{3}$ como solvente. Os espectros de ${ }^{1} \mathrm{H}$ foram referenciados utilizando-se TMS e DMF como padrões internos para efeito de comparação entre eles. Para os espectros de ${ }^{31} \mathrm{P}$, utilizou-se como referência externa $\mathrm{H}_{3} \mathrm{PO}_{4}(85 \%)$.

\section{RESULTADOS E DISCUSSÃO}

\section{Otimização da câmara fotooxidativa}

Ao realizar-se um estudo de interferentes, verificou-se que para a determinação dos teores de carbono residual por ICP OES ocorrem interferências espectrais severas no comprimento de onda 247,856 nm, devido aos elevados teores de Fe presentes. Porém, o mesmo não ocorreu na linha espectral de maior sensibilidade, $193,027 \mathrm{~nm}$, que foi utilizada sem problemas.

De modo geral, a melhor eficiência de decomposição foi obtida com a utilização da reação foto-Fenton, obtendo-se decomposições próximas a $90 \%$ em relação ao teor de carbono original. Apenas cadeias orgânicas de menor massa molar resultaram do composto orgânico inicial.

Na Figura 3 é ilustrada a superfície de respostas obtida a partir dos teores de carbono residual e das concentrações variáveis de $\mathrm{Fe}^{2+}$ e de $\mathrm{H}_{2} \mathrm{O}_{2}$ estipuladas no planejamento experimental, com tempo de exposição fixado em $24 \mathrm{~h}$. Esse período foi estabelecido após experimentos prévios demonstrarem a influência crescente do tempo na eficiência da decomposição. Com os resultados obtidos, foi possível observar que as melhores condições de decomposição do produto nas condições estudadas foram: volumes de $1 \mathrm{~mL}$ de $\mathrm{Fe}^{2+} 1000 \mathrm{mg} \mathrm{L}^{-1} \mathrm{e}$ $1,0 \mathrm{~mL}$ de $\mathrm{H}_{2} \mathrm{O}_{2} 30 \% \mathrm{~m} / \mathrm{v}$ (equivalentes a $3,6 \mathrm{mmol} \mathrm{L}^{-1}$ de $\mathrm{Fe}^{2+}$ e 1,9 mol L ${ }^{-1}$ de $\mathrm{H}_{2} \mathrm{O}_{2}$ ), para $3 \mathrm{~mL}$ de carrapaticida na diluição 1:400 v/v.

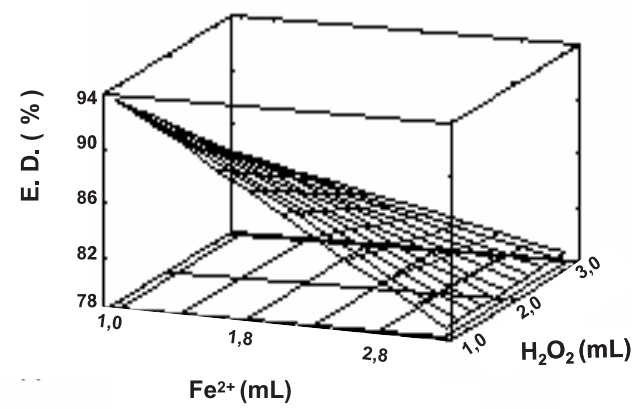

Figura 3. Gráfico de superfície de resposta do planejamento fatorial $2^{3}$, utilizado na otimização das condições experimentais para destruição de solução carrapaticida. Consideradas as variáveis volume $(\mathrm{mL})$ de $\mathrm{Fe}^{2+}(1000$ $\left.m g L^{-1}\right)$ e volume $(m L)$ de $\mathrm{H}_{2} \mathrm{O}_{2}$, quanto à eficiência de decomposição (E.D.)

No estudo de eficiência de decomposição que é apresentado na Figura 4, pode-se notar a efetiva ação da radiação UV. Quando as amostras foram irradiadas durante $24 \mathrm{~h}$, a irradiação UV possibilitou a redução em aproximadamente $50 \%$ dos teores originais de car- bono quando comparada aos estudos efetuados nas mesmas condições porém na ausência da radiação UV. Já ao se empregar a reação foto-Fenton, a radiação UV propiciou cerca de $25 \%$ de aumento da eficiência de decomposição em comparação à reação Fenton.

Os íons $\mathrm{Fe}^{3+}$ formados em função da oxidação dos íons $\mathrm{Fe}^{2+}$ são responsáveis pela formação de radicais oxidantes adicionais, promovendo maior eficiência de degradação dos compostos orgânicos em função da fotólise do ferro, formando um aquacomplexo: $\mathrm{Fe}\left(\mathrm{H}_{2} \mathrm{O}\right)_{5}(\mathrm{OH})^{2+}$ (representado como $\left.\mathrm{Fe}(\mathrm{OH})^{2+}\right)^{17}$. A fotólise do aquacomplexo regenera $\mathrm{Fe}^{2+}$, demonstrando que pouca quantidade de $\mathrm{Fe}^{2+}$ se apresenta eficiente quando submetido à radiação UV.

Por outro lado, aparentemente quando maiores volumes de reagentes são adicionados, ocorre uma diminuição na eficiência da decomposição (Figura 3). Esse fato pode estar relacionado a fatores sinérgicos e antagônicos entre essas reações competitivas pelos radicais hidroxila. Deve ser enfatizado que a concentração de equilíbrio dos íons $\mathrm{Fe}^{2+}$ e $\mathrm{Fe}^{3+}$ é fortemente dependente das condições experimentais (peróxido de hidrogênio, tipo de ligante, intensidade da luz, concentração total de íons, etc.) e mesmo a escolha de altas concentrações iniciais de $\mathrm{Fe}^{2+}$ ou $\mathrm{H}_{2} \mathrm{O}_{2}$ nem sempre proporciona efeitos benéficos ${ }^{18}$. Considerandose o ferro, quando adicionado em grandes quantidades pode competir com o carrapaticida em solução pelos radicais $\mathrm{HO} \bullet\left(\mathrm{Fe}^{2+}+\mathrm{HO} \bullet \rightarrow\right.$ $\left.\mathrm{Fe}^{3+}+\mathrm{HO}^{-}\right)^{19}$. Existe ainda o problema ambiental, uma vez que a quantidade residual dos sais de ferro adicionados gerará maior quantidade de sólidos dissolvidos. Também no caso do peróxido de hidrogênio a literatura apresenta uma grande variedade de condições, não existindo um valor ótimo. Alguns autores constataram que o aumento na concentração de peróxido não afeta a eficiência da reação de degradação de resíduos orgânicos diluídos em água, tais como ácido dicloroacético ou 2,4-diclorofenol ${ }^{18,20}$. Parra et al..$^{19}$ sugerem que pode ocorrer a autodecomposição do $\mathrm{H}_{2} \mathrm{O}_{2}$ em água e oxigênio, ou que o excesso de peróxido poderia reagir com os radicais $\mathrm{HO}$, competindo com o poluente orgânico e, conseqüentemente, diminuindo a eficiência do tratamento. É importante considerar que a maioria dos estudos é feita de forma univariável, o que pode mascarar algum efeito sinérgico que possa ocorrer. No presente experimento, o planejamento experimental realizado possibilitou, com relativamente poucos experimentos, a definição da faixa adequada de concentração de $\mathrm{Fe}^{2+}$ e $\mathrm{H}_{2} \mathrm{O}_{2}$ para a degradação do pesticida estudado, dentro das condições experimentais pré-estabelecidas (Figura 4).

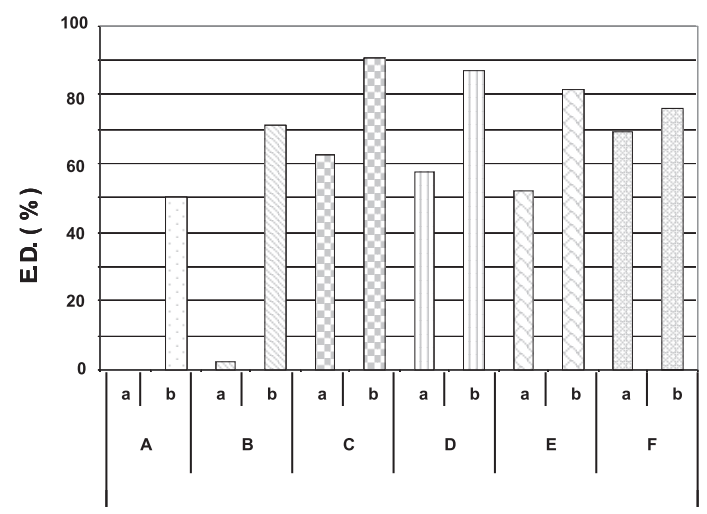

Figura 4. Eficiência de decomposição (E.D.) referente a $3 \mathrm{~mL}$ da solução carrapaticida (S.C.): $\boldsymbol{a}-\operatorname{sem} U V ; \boldsymbol{b}-\operatorname{com} U V ; \boldsymbol{A}-$ S.C.; B - S.C. $+1 \mathrm{~mL}$ $\mathrm{Fe}^{2+}\left(1000 \mathrm{mg} \mathrm{L}^{-1}\right) ; \boldsymbol{B}-$ S.C. $+1 m L \mathrm{Fe}^{2+}\left(1000 m g \mathrm{~L}^{-1}\right)+1 \mathrm{~mL} \mathrm{H}_{2} \mathrm{O}_{2} ; \boldsymbol{D}-$ S.C. $+1 m L \mathrm{Fe}^{2+}\left(1000 \mathrm{mg} \mathrm{L}^{-1}\right)+2 \mathrm{~mL} \mathrm{H}_{2} \mathrm{O}_{2} ; \boldsymbol{E}-\mathrm{S} . \mathrm{C} .+3 \mathrm{mLFe} \mathrm{F}^{2+}\left(1000 \mathrm{mg} \mathrm{L}^{-1}\right)+$ $1 \mathrm{~mL} \mathrm{H}_{2} \mathrm{O}_{2} ; \mathrm{F}-\mathrm{S} . \mathrm{C} .+3 \mathrm{~mL} \mathrm{Fe} e^{2+}\left(1000 \mathrm{mg} \mathrm{L}^{-1}\right)+3 \mathrm{~mL} \mathrm{H}_{2} \mathrm{O}_{2}$

\section{Espectros de RMN}

A partir da análise dos espectros de hidrogênio, apresentados 
Tabela 1. Valor da integração das regiões no espectro de $\mathrm{RMN}$ de ${ }^{1} \mathrm{H}$ e ${ }^{31} \mathrm{P}$

\begin{tabular}{|c|c|c|c|c|c|c|}
\hline Amostra & $\begin{array}{c}\text { Valor da } \\
\text { integração do } \\
\text { padrão interno }\end{array}$ & $\begin{array}{c}\text { Valor da } \\
\text { integração da } \\
\text { parte aromática }\end{array}$ & $\begin{array}{c}\text { Valor da integração } \\
\text { olefínica / } \\
\text { carbinólica }\end{array}$ & $\begin{array}{c}\text { Valor da } \\
\text { integração da } \\
\text { parte alifática }\end{array}$ & $\begin{array}{c}\text { Total das } \\
\text { integrações }\end{array}$ & $\begin{array}{c}\text { Presença } \\
\text { de }{ }^{31} \mathrm{P}\end{array}$ \\
\hline Pura & 1 & 6,8 & 16,9 & 39,6 & 56,5 & + \\
\hline UV & 1 & 1,7 & 11,1 & 13,7 & 26,5 & + \\
\hline $\mathrm{Fe}+\mathrm{H}_{2} \mathrm{O}_{2}$ & 1 & 2 & 7,4 & 12,1 & 21,5 & - \\
\hline $\mathrm{Fe}+\mathrm{H}_{2}^{2} \mathrm{O}_{2}^{2}+\mathrm{UV}$ & 1 & 0,7 & 7,9 & 8,1 & 16,7 & - \\
\hline
\end{tabular}

na Figura 5, é possível se comparar as integrações dos espectros da amostra pura com as demais somas dos valores das integrais das quatro amostras e verificar que a amostra que apresenta menor percentagem de material orgânico é a amostra tratada com o sistema foto-Fenton, sendo o tratamento apenas com radiação UV o que resultou em maiores teores de resíduo. Os valores de integração de cada região e sua somatória são apresentados na Tabela 1.

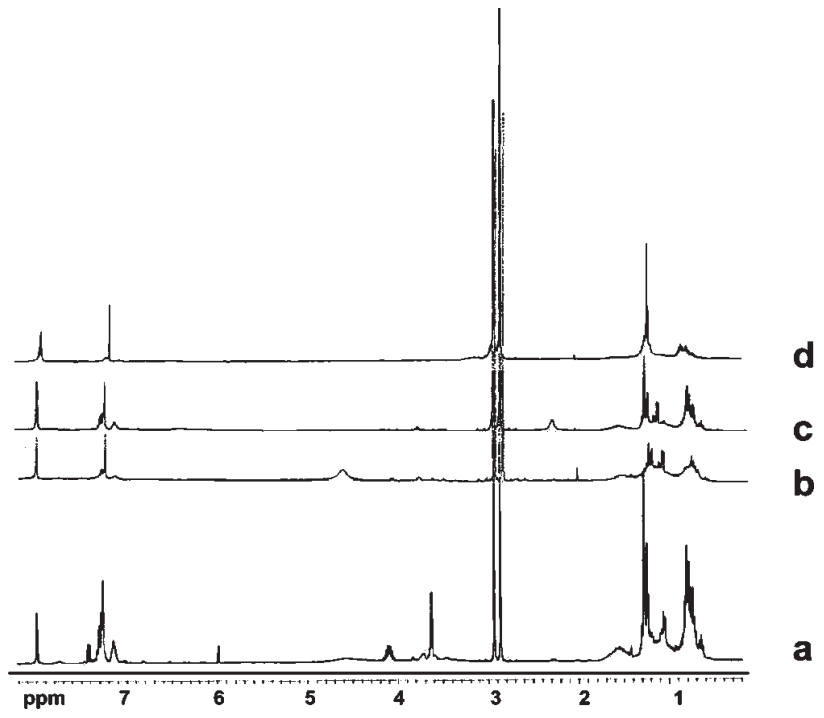

Figura 5. Espectros de $R M N^{l} H$ da solução carrapaticida: a - carrapaticida puro; $b-U V ; c-\mathrm{Fe}^{2+}+\mathrm{H}_{2} \mathrm{O}_{2} ; d-\mathrm{Fe}^{2+}+\mathrm{H}_{2} \mathrm{O}_{2}+\mathrm{UV}$

A redução mais significativa em todos os espectros foi na região aromática. Provavelmente ocorra inicialmente a ruptura do anel aromático, que é transformado em substâncias alifáticas e carbinólicas que, a seguir, são decompostas em $\mathrm{CO}_{2}$ e $\mathrm{H}_{2} \mathrm{O}$.

A presença de $\mathrm{Fe}$ paramagnético nas amostras $\mathrm{Fe}+\mathrm{UV}$ causou um alargamento excessivo das linhas no espectro de ${ }^{1} \mathrm{H}$, não sendo possível a realização de análise comparativa entre esse espectro e os demais. Vale ressaltar que a integração da região carbinólica incluindo os sinais da metila da DMF não interfere na análise dos espectros de hidrogênio, pois foram adicionadas as mesmas quantidades deste reagente $(2 \mu \mathrm{L})$ em todas as amostras.

Em relação aos testes qualitativos obtidos a partir de estudos exploratórios envolvendo $\mathrm{RMN}$ de ${ }^{31} \mathrm{P}$, foi verificada a presença desse elemento no padrão e na amostra submetida à radiação UV, sendo que nas demais amostras não foram observados sinais nos espectros obtidos sob as mesmas condições. Provavelmente a presença de ferro tenha contribuído para a formação de $\mathrm{H}_{3} \mathrm{PO}_{4}$ e/ou sais correlatos que iriam para a fase aquosa, não sendo extraídos pela fase orgânica. Os resultados demonstram a destruição dessa região do clorfenvinfos, composto presente em maior quantidade no produto estudado (Supocade ${ }^{\circledR}$ ) (Figura 2).

\section{CONCLUSÃO}

A utilização do planejamento experimental permitiu uma melhor avaliação dos dados obtidos, verificando-se que $3,6 \mathrm{mmol} \mathrm{L}^{-1}$ $\mathrm{Fe}^{2+}+1,9$ mol L $\mathrm{H}_{2} \mathrm{O}_{2}$ submetidos à radiação UV (reação fotoFenton) permitem a destruição de $94 \%$ do composto orgânico original, sendo essas as condições que apresentaram maior eficiência de decomposição entre as alternativas avaliadas. A proposta apresenta-se viável e potencialmente adequada para tratamento de resíduos tóxicos que contenham compostos orgânicos solúveis, além de poder ser utilizada diretamente no campo, sem necessidade de transporte até o laboratório. O mesmo protótipo já foi aplicado para tratamento de resíduos fenólicos e nitrofenólicos, sendo obtidos resultados bastante promissores.

\section{AGRADECIMENTOS}

À FAPESP, ao CNPq e à EMBRAPA pelo suporte financeiro e bolsas concedidas.

\section{REFERÊNCIAS}

1. Nogueira, R. F. P.; Jardim, W. F. A.; Quim. Nova 1998, 21, 69.

2. Trovó, A. G.; Villa, R. D.; Nogueira, R. F. P.; Quim. Nova 2005, $28,847$.

3. De Laat, J.; Gallard, H.; Ancelin, S.; Legube, B.; Chemosphere 1999, 39, 2693.

4. Xu, Y.; Chemosphere 2001, 43, 1103.

5. Naffrechoux, E.; Chanous, S.; Petrier, C.; Suptil, J.; Ultrason. Sonochem. 2000, 7, 255 .

6. Teixeira, C. P. A. B.; Introdução a conceitos teóricos e práticos sobre processos oxidativos avançados, I. Escola de Química Ambiental do IQUNICAMP, 2004.

7. Lu, M. C.; Roam, G. D.; Chen, J. N.; Huang, C. P.; Water Res. 1994, 30, 29.

8. Pignatello, J. J.; Environ. Sci. Technol. 1992, 26, 944.

9. Zepp, R. G.; Faust, B. C.; Hoigné, J.; Environ. Sci. Technol. 1992, 26, 313.

10. Gernjak, W.; Krutzler, T.; Glaser, A.; Malato, S.; Caceres, J.; Bauer, R.; Fernández-Alba, A. R.; Chemosphere 2003, 50, 71.

11. Pereira-Filho, E. R.; Poppi, R. J.; Arruda, M. A. Z.; Quim. Nova 2002, 25, 246.

12. Neto, B. B.; Scarminio, I. S.; Bruns, R. E.; Como fazer experimentos pesquisa e desenvolvimento na ciência e na indústria, Ed. Unicamp: Campinas, 2001.

13. Zhou, C. Y.; Wong, M. K.; Koh, L. L.; Wee, Y. C.; Anal. Chim. Acta 1995, 314, 121.

14. Martín-Garcia, M. B.; Bellido-Milla, D.; Jimenez-Jimenez, A.; HernándezArtiga, M. P.; Fresenius J. Anal. Chem. 1999, 364, 527.

15. Ferreira, S. L. C.; dos Santos, W. N. L.; Bezerra, M. A.; Lemos, V. A.; Bosque-Sendra, J. M.; Anal. Bioanal. Chem. 2003, 375, 443.

16. Gouveia, S. T.; Silva, F. V.; Costa, L. M.; Nogueira, A. R. A.; Nóbrega, J. A.; Anal. Chim. Acta 2001, 445, 269.

17. Katsumata, H.; Kaneco, S.; Suzuki T.; Ohta, K.; Yobiko, Y.; Chem. Eng. J. 2005, 108, 269.

18. Gogate, P. R.; Pandit, A. B.; Adv. Environ. Res. 2004, 8, 553.

19. Parra, S.; Sarria, V.; Malato, S.; Péringer, P.; Pulgarin, C.; Appl. Catal., B 2000, 27, 153.

20. Nogueira, R. F. P.; Guimarães, J. R.; Water Res. 2000, 34, 895. 